\title{
Long Time Behaviour of an Infinite Particle System
}

\section{Luen-Chau Li}

Department of Mathematics, Pennsylvania State University, University Park, PA 16802, USA

Abstract. The long time behaviour of the semi-infinite Toda lattice is deduced from a set of identities for the squared eigenfunctions of the Toda flow.

\section{Introduction}

Let $\ell_{2}^{+}$be the real Hilbert space of sequences $u=\left(u_{1}, u_{2}, \ldots\right), u_{i} \in \mathbb{R}$ such that $\|u\|^{2}$ $=\sum_{i=1}^{\infty} u_{i}^{2}<\infty$, and let $D=\left\{u \in \ell_{2}^{+}: u_{i} \neq 0\right.$ for finitely many $\left.i\right\}$. Recently, the study of the semi-infinite Toda lattice [3] has led us to consider isospectral flows on bounded symmetric operators $L$ in $\ell_{2}^{+}$. More precisely, if $L_{0}$ is a bounded self-adjoint operator on $\ell_{2}^{+}$, then, by extending the well-known $Q R$ decomposition for matrices to the present case, one has

$$
e^{t L_{0}}=Q(t) R(t), \quad t \in \mathbb{R},
$$

where $Q(t)$ is orthogonal $\left(Q^{T}(t) Q(t)=Q(t) Q^{T}(t)=I\right)$ and $R(t)$ is upper triangular $\left[R_{i j}(t)=0\right.$ for $\left.i>j\right]$ with $R_{i i}(t)>0$. Clearly, the map $L_{0} \rightarrow \phi\left(t, L_{0}\right) \equiv L(t)$ $\equiv Q^{T}(t) L_{0} Q(t)$ defines an isospectral flow on bounded symmetric operators on $\ell_{2}^{+}$, and we shall refer to it as the Toda flow.

In [1], we develop new techniques to study various properties of the isospectral flows which are related to the Toda flow. In particular, we have a dynamical version of the min-max theorem from which we derive the following

Theorem. If $L_{0}$ is a Jacobi operator ${ }^{1}$, then for all $t, \phi\left(t, L_{0}\right)$ is also a Jacobi operator. Moreover, as $t \rightarrow \infty, \phi\left(t, L_{0}\right)$ converges strongly to a diagonal operator $A=\operatorname{diag}\left(\alpha_{1}, \alpha_{2}, \ldots\right)$, where

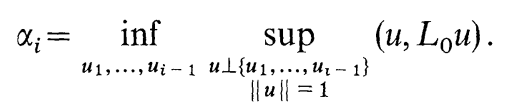

\footnotetext{
${ }^{1}$ A Jacobi operator is a real, bounded, symmetric, tridiagonal operator in $\ell_{2}^{+}$with strictly positive off-diagonal entries
} 
From the well-known connection between the Toda flow on Jacobi operators and the semi-infinite Toda lattice via the Flaschka transformation [2], i.e.

$$
L(t)_{i i}=-\frac{1}{2} y_{i}(t), \quad L(t)_{i, i+1}=\frac{1}{2} \exp \left[\left(x_{i}(t)-x_{i+1}(t)\right) / 2\right], \quad i=1,2, \ldots
$$

$\left[x_{i}(t), y_{i}(t)\right.$ are respectively the position and the momentum of the $i^{\text {th }}$ particle of the lattice at time $t]$, we conclude that as $t \rightarrow \infty$, the particles are moving away from each other, as in the finite particle system of Moser [4]. Moreover, the $i^{\text {th }}$ particle, say, is moving with an asymptotic momentum given by $y_{i}(\infty)=-2 \alpha_{i}$.

It is the purpose of this paper to give a simple proof of the above result making full use of the tridiagonality of the operators involved. The key of our proof is an interesting set of identities (see Sect. 3, Proposition 1) which give the rate of change of some combination of the $\psi^{2}(k, \lambda, t)$ 's under the Toda flow, where $\psi(\lambda, t)$ $=(\psi(1, \lambda, t), \psi(2, \lambda, t), \ldots)$ is a generalized eigenvector of $L(t)$, suitably normalized. Making use of these identities, a relation between the entries of the limiting operator $A$ and the support of the spectral measure of $L_{0}$ is derived, and the theorem follows as an easy consequence.

\section{Preliminaries}

We first collect a number of facts which are extensions of well-known results in the matrix case (see, e.g. [6]). For the proof, we refer the reader to [1].

Lemma 1 ( $Q R$ decomposition). Let $P$ be a bounded, invertible operator on $\ell_{2}^{+}$. We have the unique decomposition

$$
P=Q R,
$$

where $Q$ is orthogonal and $R$ is a bounded invertible upper triangular operator with $R_{i i}>0$.

Proposition 1. Let $L_{0}$ be a bounded self-adjoint operator on $\ell_{2}^{+}$and let

$$
e^{t L_{0}}=Q(t) R(t)
$$

be the $Q R$ decomposition of $e^{t L_{0}}$. Then the map

$$
L_{0} \rightarrow \phi\left(t, L_{0}\right) \equiv L(t) \equiv Q^{T}(t) L_{0} Q(t)
$$

defines a flow on the space of bounded symmetric operators and solves the initial value problem

$$
\begin{aligned}
\dot{L}=[B, L] & =B L-L B, L(0)=L_{0}, \\
B & =L_{+}-L_{-},{ }^{2}
\end{aligned}
$$

in the weak sense on $D$, i.e., $(u, L v)^{\circ}=-(B u, L v)-(L u, B v)$ for all $u, v \in D$. Furthermore, if $L_{0}$ is a Jacobi operator, then $L(t)$ is a Jacobi operator for all $t$ and is the unique weak solution to (*).

By modifying an argument of Moser [4], we also obtain

\footnotetext{
${ }^{2} L_{+}$and $L_{-}$are the strict upper projection and lower projection of $L$ respectively
} 
Proposition 2. Suppose $L(t)$ is the (weak) solution of the Toda equation $\dot{L}=[B, L]$, $L(0)=L_{0}$. Then $L(t)$ converges strongly to a diagonal operator $A=\operatorname{diag}\left(\alpha_{1}, \alpha_{2}, \ldots\right)$ as $t \rightarrow \infty$ with $\alpha_{i} \in \sigma\left(L_{0}\right)$. Moreover, if $L_{0}$ is a Jacobi operator, then $\alpha_{1} \geqq \alpha_{2} \geqq \alpha_{3} \ldots$

Remark. Proposition 2 is a preliminary result on the long time behaviour of the infinite particle system. However, in contrast to the matrix case considered by Moser, this does not automatically give the precise values of the $\alpha_{i}$ 's. This is because for operators, strong convergence is weaker than norm convergence, and the spectrum shrinks in our case.

We now summarize the spectral theory of Jacobi operators which we will need. Let $L=\int \lambda d E_{\lambda}$ be a Jacobi operator on $\ell_{2}^{+}$and $\mu(\Delta)=\mu(\Delta ; L) \equiv\left(e_{1}, E(\Delta) e_{1}\right)$. Then $\mu$ is a spectral measure for $L$ which is compactly supported in $\mathbb{R}$ and of mass 1 . Moreover, it is well-known that the map $L \rightarrow \mu(\Delta ; L)$ is bijective. Associated with $L$ is a sequence of polynomials $P(\lambda)=(P(1, \lambda), P(2, \lambda), \ldots)$ obeying $(L-\lambda) P=0$ with $P(1, \lambda) \equiv 1$. Using these polynomials, we define the Fourier transform

then

$$
(F u)(\lambda)=\sum_{k=1}^{\infty} P(k, \lambda) u(k), \quad u \in D
$$

$$
\|u\|^{2}=\int(F u)^{2}(\lambda) d \mu(\lambda)=\|F u\|_{L_{2}(d \mu)}^{2},
$$

and $F$ extends to an orthogonal map from $\ell_{2}^{+}$to $L_{2}(d \mu)$. Finally, $L$ is diagonalized by $F$, i.e.

$$
(F L u)(\lambda)=\lambda(F u)(\lambda)
$$

\section{Proof of the Theorem}

Throughout this section, $L_{0}$ is a fixed Jacobi operator with spectral measure $d \mu$, and $L_{0} \rightarrow L(t) \equiv Q^{T}(t) L_{0} Q(t)$ is the Toda flow. Set

$$
\lambda_{i}=\inf _{u_{1}, \ldots, u_{2-1}} \sup _{\substack{u \perp\left\{u_{1}, \ldots, u_{2}\right\} \\\|u\|=1}}\left(u, L_{0} u\right),
$$

and write $a_{i}(t) \equiv\left(e_{i}, L(t) e_{i}\right), b_{i}(t)=\left(e_{i}, L(t) e_{i+1}\right)$. From Proposition 2, Sect. 2, $s$ $\lim _{t \rightarrow \infty} L(t)=A=\operatorname{diag}\left(\alpha_{1}, \alpha_{2}, \ldots\right)$, we now show $\alpha_{n}=\lambda_{n}$ for all $n$.

Lemma 1. For all $n, \alpha_{n} \leqq \lambda_{n}$.

Proof. Let $P_{n}$ be the projection operator onto the $n$-dimensional subspace $E_{n}$ $=\operatorname{span}\left(e_{1}, e_{2}, \ldots, e_{n}\right)$ and $\alpha_{n}(t)$ the smallest eigenvalue of $P_{n} L(t) P_{n} \mid E_{n}$. By min-max,

$$
\begin{aligned}
\alpha_{n}(t) & =\inf _{u_{1}, \ldots, u_{n-1} \in E_{n}} \sup _{\substack{u \in E_{n}, u \perp\left\{u_{1}, \ldots, u_{n-1}\right\} \\
\|\boldsymbol{u}\|=1}}\left(u, P_{n} L(t) P_{n} u\right) \\
& =\inf _{u_{1}, \ldots, u_{n-1}} \sup _{\substack{u \in E_{n}, u \perp\left\{\boldsymbol{P}_{n} u_{1}, \ldots, P_{n} u_{n-1}\right\} \\
\|u\|=1}}(u, L(t) u) \\
& \leqq \inf _{u_{1}, \ldots, u_{n-1}} \sup _{\substack{u \perp\left\{u_{1}, \ldots, u_{n-1}\right\} \\
\|\boldsymbol{u}\|=1}}(u, L(t) u) \\
& =\lambda_{n} .
\end{aligned}
$$

The assertion now follows from the observation that $\alpha_{n}(t) \rightarrow \alpha_{n}$ as $t \rightarrow \infty$. 
Lemma 2. Under the Toda flow,

(a) $d \mu(\lambda, t)=\frac{e^{2 \lambda t} d \mu(\lambda)}{\int e^{2 \lambda^{\prime} t} d \mu\left(\lambda^{\prime}\right)}$.

(b) $a_{1}(t)=\frac{\int \lambda e^{2 \lambda t} d \mu(\lambda)}{\int e^{2 \lambda t} d \mu(\lambda)}$.

(c) $\dot{P}(k, \lambda, t)=-b_{k-1}(t) P(k-1, \lambda, t)+b_{k}(t) P(k+1, \lambda, t)$ $+\left(a_{1}(t)-\lambda\right) P(k, \lambda, t), \quad k=1,2, \ldots,\left(b_{0}(t) \equiv 0\right)$.

(d) Let $\psi(k, \lambda, t)=\frac{e^{\lambda t} P(k, \lambda, t)}{\left(\int e^{2 \lambda^{\prime} t} d \mu\left(\lambda^{\prime}\right)\right)^{1 / 2}}$, then $\dot{\psi}(k, \lambda, t)=-b_{k-1}(t) \psi(k-1, \lambda, t)+b_{k}(t) \psi(k+1, \lambda, t), \quad k=1,2, \ldots$.

Proof. (a) It is sufficient to show that

$$
\left(e_{1},(z-L(t))^{-1} e_{1}\right)=\frac{\int(z-\lambda)^{-1} e^{2 \lambda t} d \mu(\lambda)}{\int e^{2 \lambda t} d \mu(\lambda)} \text { for all } z \notin \sigma\left(L_{0}\right) .
$$

But from the definition of $L(t) \equiv Q^{T}(t) L_{0} Q(t)$ and the relation

we have

$$
Q(t) e_{1}=\frac{e^{t L_{0}} e_{1}}{\left\|e^{t L_{0}} e_{1}\right\|}
$$

$$
\left(e_{1},(z-L(t))^{-1} e_{1}\right)=\left(Q(t) e_{1},\left(z-L_{0}\right)^{-1} Q(t) e_{1}\right)
$$

as required.

$$
=\frac{\left(e^{t L_{0}} e_{1},\left(z-L_{0}\right)^{-1} e^{t L_{0}} e_{1}\right)}{\left\|e^{t L_{0}} e_{1}\right\|^{2}}=\frac{\int(z-\lambda)^{-1} e^{2 \lambda t} d \mu(\lambda)}{\int e^{2 \lambda t} d \mu(\lambda)},
$$

(b) $a_{1}(t)=\left(e_{1}, L(t) e_{1}\right)=\left(Q(t) e_{1}, L_{0} Q(t) e_{1}\right)$

$$
=\frac{\left(e^{t L_{0}} e_{1}, L_{0} e^{t L_{0}} e_{1}\right)}{\left\|e^{t L_{0}} e_{1}\right\|^{2}}=\frac{\int \lambda e^{2 \lambda t} d \mu(\lambda)}{\int e^{2 \lambda t} d \mu(\lambda)} .
$$

(c) Standard calculation shows that

$$
\dot{P}(k, \lambda, t)=-b_{k-1}(t) P(k-1, \lambda, t)+b_{k}(t) P(k+1, \lambda, t)+C(\lambda, t) P(k, \lambda, t) .
$$

The function $C(\lambda, t)$ is then determined from the normalization $P(1, \lambda, t) \equiv 1$.

(d) By direct differentiation, making use of (b) and (c), we obtain

$$
\begin{aligned}
\dot{\psi}(k, \lambda, t) & =\frac{e^{\lambda t} \dot{P}(k, \lambda, t)}{\left(\int e^{2 \lambda^{\prime} t} d \mu\left(\lambda^{\prime}\right)\right)^{1 / 2}}+\frac{e^{\lambda t} P(k, \lambda, t)}{\left(\int e^{2 \lambda^{\prime} t} d \mu\left(\lambda^{\prime}\right)\right)^{1 / 2}}\left(\lambda-a_{1}(t)\right) \\
& =-b_{k-1}(t) \psi(k-1, \lambda, t)+b_{k}(t) \psi(k+1, \lambda, t) .
\end{aligned}
$$

Remark. The functions $\psi(1, \lambda, t), \psi(2, \lambda, t), \ldots$ are obtained by applying GramSchmidt to the vectors $e^{\lambda t}, \lambda e^{\lambda t}, \lambda^{2} e^{\lambda t}, \ldots$ in $L_{2}(d \mu)$. In particular, we have

$$
\int \psi^{2}(k, \lambda, t) d \mu(\lambda)=1 \text {. }
$$

Proposition 1. Under the Toda flow,

$$
\begin{gathered}
\left(\psi^{2}(1, \lambda, t)\right)^{\cdot}=2\left(\lambda-a_{1}(t)\right) \psi^{2}(1, \lambda, t) . \\
\left(\psi^{2}(k, \lambda, t)+2 \sum_{j=1}^{k-1} \psi^{2}(j, \lambda, t)\right)^{\cdot}=2\left(\lambda-a_{k}(t)\right) \psi^{2}(k, \lambda, t), \quad k \geqq 2 .
\end{gathered}
$$


Proof. By Lemma 2(d) and the recurrence relation $b_{1}(t) \psi(2, \lambda, t)$ $=\left(\lambda-a_{1}(t)\right) \psi(1, \lambda, t)$, we clearly have

$$
\begin{aligned}
\left(\psi^{2}(1, \lambda, t)\right)^{\circ} & =2 \psi(1, \lambda, t) \dot{\psi}(1, \lambda, t) \\
& =2 b_{1}(t) \psi(1, \lambda, t) \psi(2, \lambda, t)=2\left(\lambda-a_{1}(t)\right) \psi^{2}(1, \lambda, t) .
\end{aligned}
$$

For $k \geqq 2$, use of Lemma 2(d) yields

$$
\begin{aligned}
\left(\psi^{2}(k, \lambda, t)+2 \sum_{j=1}^{k-1} \psi^{2}(j, \lambda, t)\right)^{\cdot}= & 2 \psi(k, \lambda, t)\left(-b_{k-1}(t) \psi(k-1, \lambda, t)\right. \\
& \left.+b_{k}(t) \psi(k+1, \lambda, t)\right) \\
& +4 \sum_{j=1}^{k-1} \psi(j, \lambda, t)\left(-b_{j-1}(t) \psi(j-1, \lambda, t)\right. \\
& \left.+b_{j}(t) \psi(j+1, \lambda, t)\right) \\
= & 2 \psi(k, \lambda, t)\left(-b_{k-1}(t) \psi(k-1, \lambda, t)\right. \\
& \left.+b_{k}(t) \psi(k+1, \lambda, t)\right) \\
& +4 b_{k-1}(t) \psi(k-1, \lambda, t) \psi(k, \lambda, t) \\
= & 2 \psi(k, \lambda, t)\left(b_{k-1}(t) \psi(k-1, \lambda, t)+b_{k}(t) \psi(k+1, \lambda, t)\right) .
\end{aligned}
$$

But $b_{k-1}(t) \psi(k-1, \lambda, t)+b_{k}(t) \psi(k+1, \lambda, t)=\left(\lambda-a_{k}(t)\right) \psi(k, \lambda, t)$, therefore,

$$
\left(\psi^{2}(k, \lambda, t)+2 \sum_{j=1}^{k-1} \psi^{2}(j, \lambda, t)\right)^{\cdot}=2\left(\lambda-a_{k}(t)\right) \psi^{2}(k, \lambda, t) .
$$

Proposition 2. If $\alpha_{k+1}<\alpha_{k}$, then $\mu\left(\left(\alpha_{k+1}, \alpha_{k}\right)\right)=0$.

Proof. Suppose the contrary, then we can find an open interval $\Delta=(r, s)$ sitting inside $\left(\alpha_{k+1}, \alpha_{k}\right)$ such that $\int_{\Delta} d \mu(\lambda) \neq 0$. Let $\varepsilon=\min \left(\frac{1}{2}\left(r-\alpha_{k+1}\right), \frac{1}{2}\left(\alpha_{k}-s\right)\right)$ and choose
$T>0$ so that

$$
\min \left(r-a_{k+1}(t), a_{k}(t)-s\right) \geqq \varepsilon, \quad t \geqq T .
$$

For $\lambda \in \Delta$, we then have

$$
\min \left(\lambda-a_{k+1}(t), a_{k}(t)-\lambda\right) \geqq \varepsilon, \quad t \geqq T .
$$

Now, invoke the identity in Proposition 1, we find

$$
\left(\psi^{2}(k+1, \lambda, t)+2 \sum_{j=1}^{k} \psi^{2}(j, \lambda, t)\right)^{\cdot}=2\left(\lambda-a_{k+1}(t)\right) \psi^{2}(k+1, \lambda, t)
$$

$$
\text { and hence } \quad \geqq 2 \varepsilon \psi^{2}(k+1, \lambda, t) \geqq 0, \quad \lambda \in \Delta, t \geqq T \text {, }
$$

$$
\psi^{2}(k+1, \lambda, t)+2 \sum_{j=1}^{k} \psi^{2}(j, \lambda, t) \geqq \psi^{2}(k+1, \lambda, T)+2 \sum_{j=1}^{k} \psi^{2}(j, \lambda, T)>0,
$$

In particular, this implies

$$
\lambda \in \Delta, t \geqq T .
$$

$$
\begin{aligned}
& \int_{\Delta} \psi^{2}(k+1, \lambda, t) d \mu(\lambda)+2 \sum_{j=1}^{k} \int_{\Delta} \psi^{2}(j, \lambda, t) d \mu(\lambda) \\
& \geqq \int_{\Delta} \psi^{2}(k+1, \lambda, T) d \mu(\lambda)+2 \sum_{j=1}^{k} \int_{\Delta} \psi^{2}(j, \lambda, T) d \mu(\lambda)>0, \quad t \geqq T .
\end{aligned}
$$


Meanwhile, from the recurrence relation

$$
\left(\lambda-a_{k+1}(t)\right) \psi(k+1, \lambda, t)=b_{k}(t) \psi(k, \lambda, t)+b_{k+1}(t) \psi(k+2, \lambda, t),
$$

we obtain

$$
\left|\int_{\Delta}\left(\lambda-a_{k+1}(t)\right) \psi^{2}(k+1, \lambda, t) d \mu(\lambda)\right| \leqq b_{k}(t)+b_{k+1}(t) \rightarrow 0 \quad \text { as } \quad t \rightarrow \infty
$$

But for $t \geqq T$,

$$
\int_{\Delta}\left(\lambda-a_{k+1}(t)\right) \psi^{2}(k+1, \lambda, t) d \mu(\lambda) \geqq \varepsilon \int_{\Delta} \psi^{2}(k+1, \lambda, t) d \mu(\lambda) .
$$

Therefore,

$$
\lim _{t \rightarrow \infty} \int_{\Delta} \psi^{2}(k+1, \lambda, t) d \mu(\lambda)=0
$$

In a similar way, we can show

$$
\lim _{t \rightarrow \infty} \int_{\Delta} \psi^{2}(j, \lambda, t) d \mu(\lambda)=0, \quad j=1, \ldots, k
$$

We thus arrive at a contradiction when we let $t \rightarrow \infty$ in $(*)$. Hence we must have $\mu\left(\left(\alpha_{k+1}, \alpha_{k}\right)\right)=0$.

Theorem. $\alpha_{n}=\lambda_{n}$ for all $n$.

Proof. Using Lemma 2(b), we find that as $t \rightarrow \infty$,

$$
a_{1}(t)=\frac{\int \lambda e^{2 \lambda t} d \mu(\lambda)}{\int e^{2 \lambda t} d \mu(\lambda)} \rightarrow \sup (\operatorname{supp} \mu)=\lambda_{1}
$$

Therefore, $\alpha_{1}=\lambda_{1}$. From the min-max principle (see e.g. [5]), we have either:

(a) $\lambda_{1}=\sup \sigma_{\text {ess }}\left(L_{0}\right)$ in which case $\lambda_{1}=\lambda_{2}=\ldots$

or

(b) $\lambda_{2}<\lambda_{1}$.

Consider first case (a). By Lemma $1, \alpha_{2} \leqq \lambda_{1}$. If we had $\alpha_{2}<\lambda_{1}$, then according to Proposition 2, we would have $\mu\left(\left(\alpha_{2}, \alpha_{1}\right)\right)=0$. But this is impossible as $\lambda_{1}=\sup \sigma_{\text {ess }}\left(L_{0}\right)$. Therefore, $\alpha_{2}=\lambda_{1}$ and indeed all the $\alpha_{n}$ 's are equal to $\lambda_{1}$ in this case. In case (b), if we had $\alpha_{2}<\lambda_{2}$, then again we would obtain $\mu\left(\left(\alpha_{2}, \alpha_{1}\right)\right)=0$ by Proposition 2 which is contradictory to $\lambda_{2} \in\left(\alpha_{2}, \alpha_{1}\right)$. So we must have $\alpha_{2}=\lambda_{2}$. The proof now proceeds inductively.

Acknowledgement. The author would like to thank Professor P. Deift for helpful conversations and encouragement. 


\section{References}

1. Deift, P., Li, L.-C., Tomei, C.: Toda flows with infinitely many variables. J. Funct. Anal. 64, 358-402 (1985)

2. Flaschka, H.: The Toda lattice. I. Phys. Rev. B 9, 1924-1925 (1974)

3. Li, L.-C.: Ph. D. Thesis. New York University 1983

4. Moser, J.: Finitely many mass points on the line under the influence of an exponential potentialAn integrable system, dynamical systems, theory and applications. Moser, J. (ed.), Berlin, Heidelberg, New York: Springer 1975, pp. 467-497

5. Reed, M., Simon, B.: Methods of modern mathematical physics. IV. Analysis of operators. New York: Academic Press 1978

6. Symes, W.: Hamiltonian group actions and integrable systems. Physica D 10, 339-374 (1980)

Communicated by O. E. Lanford

Received October 26, 1984; in revised form February 27, 1987 
